\title{
The simulation and prediction of spatio - temporal urban growth trends using cellular automata models: a review
}

\begin{abstract}
In recent years, several types of simulation and prediction models have been used within a GIS environment to determine a realistic future for urban growth patterns. These models include quantitative and spatio-temporal techniques that are implemented to monitor urban growth. The results derived through these techniques are used to create future policies that take into account sustainable development and the demands of future generations. The aim of this paper is to provide a basis for a literature review of urban Cellular Automata (CA) models to find the most suitable approach for a realistic simulation of land use changes. The general characteristics of simulation models of urban growth and urban CA models are described, and the different techniques used in the design of these models are classified. The strengths and weaknesses of the various models are identified based on the analysis and discussion of the characteristics of these models. The results of the review confirm that the CA model is one of the strongest models for simulating urban growth patterns owing to its structure, simplicity, and possibility of evolution. Limitations of the CA model, namely weaknesses in the quantitative aspect, and the inability to include the driving forces of urban growth in the simulation process, may be minimized by integrating it with other quantitative models, such as via the Analytic Hierarchy Process (AHP), Markov Chain and frequency ratio models. Realistic simulation can be achieved when socioeconomic factors and spatial and temporal dimensions are integrated in the simulation process.
\end{abstract}

Keyword: Simulation; Prediction; Urban growth; Spatio-temporal; Cellular automata 